\title{
A natural macaque model of Bardet-Biedl appears in Oregon
}

Peterson, S.M. et al. Exp. Eye Res. 189, 107825 (2019)

Lining the back of the healthy primate eye-be it human or rhesus macaque-you should find a retina with fovea and macula. So when a pair of postmortem eyes from a 4-year old male raised at the Oregon National Primate Research Center (ONPRC) turned up one day last year without the necessary photoreceptor cells for sight, it was a notable occasion for Martha Neuringer.

Neuringer is a researcher at the Oregon Health and Science University in Portland who studies degenerative diseases of the retina. She had spent over twenty years looking for just such animals amongst the natural, outbred colony living at the ONPRC - to no avail. In the meantime, there were nonhuman primate studies to be done to develop and improve surgical techniques and the gene delivery methods, such as AAV vectors, to ferry eventual treatments into humans with various retinal diseases-even though the animals themselves lacked a genetic mutation to be corrected for. "We've done a number of those kinds of studies that have been part of the process leading to human clinical trials," she says, "But we haven't had a model where we can test the efficacy of the treatmentdoes it cure or ameliorate the disease?" She and other colleagues had been exploring the possibility of using CRISPR editing in macaques to jumpstart that quest for a genetic nonhuman primate model of retinal degeneration... when the first natural one finally turned up.

And then there were two. Shortly thereafter, a sibling of that first animal fell ill with kidney failure; Neuringer and her colleagues were able to confirm retinal degeneration before the animal was euthanized. "We knew we had two siblings with the same degeneration," says Neuringer, "We immediately let our primate genetics group know."

It was off to the genome. In a matter of just eight days-blindness plus renal failure narrowed the options considerably, says Neuringer-the mutation responsible for the pairs' retinal degeneration was revealed.

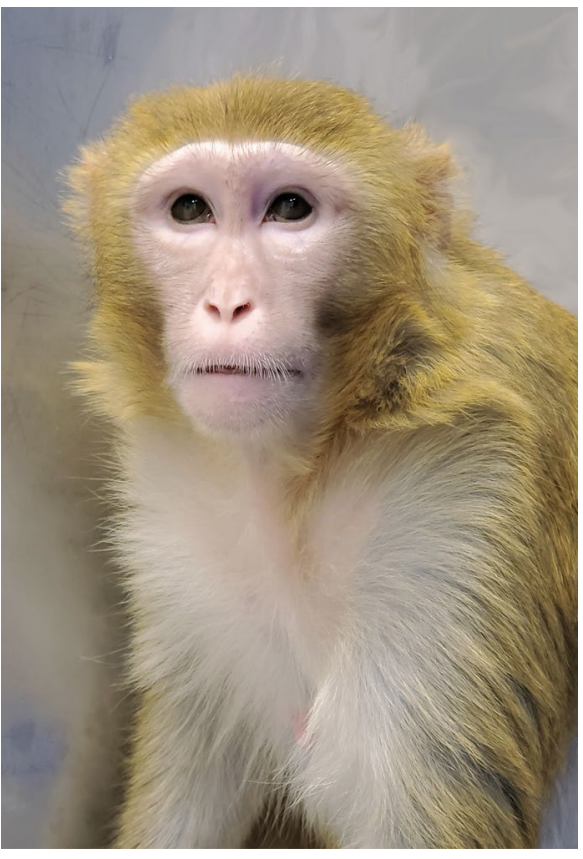

The third macaque identified with retinal degeneration due a mutation in BBS7, a gene implicated in Bardet-Biedl syndrome. Credit: Oregon Health and Science University

It involved a frameshift variant in the gene $B B S 7$, one of several that contribute to Bardet-Biedl syndrome (BBS), a ciliopathy that leads to photoreceptor degeneration and blindness as well as kidney dysfunction, polydactyly, hypogonadism, and obesity.

All of the animals at the ONPRC are carefully pedigreed, and 2000 of the macaques have had their genomes sequenced (that data is made available through The ONPRC Macaque Genotype and Phenotype Resource). Among the larger colony, 40 living animals were identified as carriers of the BBS7 c.160delG variant, along with a third-younger-homozygous animal. At three and a half years old (roughly equivalent to a 14 year old human), her condition had not yet declined to the extent of her half siblings says Neuringer, but initial evaluations revealed signs of retinal degeneration, most severely in the macula. The care and research staff at the ONPRC will be following her closely in coming years.

The syndrome is rare, affecting only about 1 in 140,000 to 160,000 newborns in North America and Europe; as such, information about its progression is limited and there is currently no cure available. Animal to animal, the trio of macaques examined so far do lack some of the other characteristic phenotypes of BBS, such as polydactyly and consistent obesity, but Neuringer says it's a thrilling find nonetheless-after so many years of screening, they now have a nonhuman primate model of an inherited retinal degenerative disease with a known gene mutation to study.

The challenge going forward will be to make more animals says Neuringer, to better understand the progression of the disease and its variable phenotypes and to eventually test both gene and cell replacement therapies. Neuringer says she has redirected supplemental funding she had received from the nonprofit group Research to Prevent Blindness from making CRISPR-edited models to breeding those natural carriers; an additional grant from the National Eye Institute's Audacious Goals Initiative awarded in August will support that propagation effort in Oregon as well.

Though Bardet-Biedl is just one genetic form of degenerative blindness, Neuringer is hopeful there will be lessons from these animals that might be applicable to other disorders as well, such as retinitis pigmentosa, an inherited condition that occurs in 1 in 4000 people. The experience of developing cell replacement therapies for this particular disease should generalize to other forms of photoreceptor degeneration and make the process more rapid for others, she says.

\section{Ellen P. Neff}

Published online: 16 December 2019

https://doi.org/10.1038/s41684-019-0449-9 